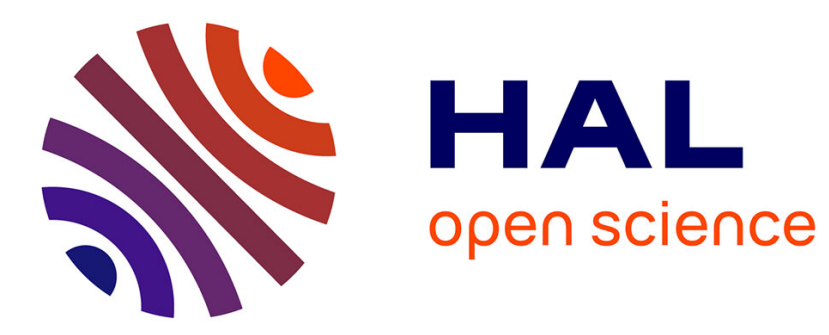

\title{
Aptitude de plusieurs déchets urbains à fournir du phosphore aux cultures
}

Bernard Pommel, Michel Lasserre

\section{To cite this version:}

Bernard Pommel, Michel Lasserre. Aptitude de plusieurs déchets urbains à fournir du phosphore aux cultures. Agronomie, 1982, 2 (9), pp.851-857. hal-00884455

\section{HAL Id: hal-00884455 \\ https://hal.science/hal-00884455}

Submitted on 1 Jan 1982

HAL is a multi-disciplinary open access archive for the deposit and dissemination of scientific research documents, whether they are published or not. The documents may come from teaching and research institutions in France or abroad, or from public or private research centers.
L'archive ouverte pluridisciplinaire HAL, est destinée au dépôt et à la diffusion de documents scientifiques de niveau recherche, publiés ou non, émanant des établissements d'enseignement et de recherche français ou étrangers, des laboratoires publics ou privés. 


\title{
Aptitude de plusieurs déchets urbains à fournir du phosphore aux cultures
}

\author{
Bernard POMMEL, \\ avec la collaboration technique de Michel LASSERRE \\ I.N.R.A., Station d'Agronomie, Centre de Recherches de Bordeaux, F 33140 Pont-de-la-Maye.
}

\section{RÉSUMÉ \\ Boue résiduaire, Compost d'ordures ménagères, Phosphore, Assimilabilité, Fermentescibilité, Extractant chimique, Modele mathématique, Cinétique.}

On a caractérisé 5 boues résiduaires et un compost d'ordures ménagères, dont on a mesuré la biodégradabilité et desquels on a cherché à extraire le phosphore par des réactifs chimiques plus ou moins agressifs.

On a également suivi la cinétique d'extraction, par du ray-grass, du phosphore de ces mêmes produits et des scorics Thomas, en utilisant le phosphate monocalcique comme fertilisant de référence.

Par comparaison à l'effet du phosphate monocalcique, l'efficience relative du phosphore des déchets urbains est décrite par une expression du type $E=k^{c}$ qui rend compte de plus de 98 p. 100 des variations où : E représente l'efficience relative, en p. cent,

t, le temps de contact entre les racines et la source de phosphore à l'intérieur de certaines limites,

$k$ et $c$ étant des coefficients propres à chaque déchet organique.

Les boues compostées avec de la sciure sont les plus rapides à céder leur phosphore à la plante, suivies par les boues physicochimiques; le chaulage des boues digérées n'a pas sensiblement modifié leur comportement. Enfin, la plante n'utilise que très lentement le phosphore des ordures ménagères.

Nous n'avons trouvé aucune relation d'utilisation commode entre la fermentiscibilité des ordures, le phosphore extrait par divers réactifs et celui extrait par la plante.

\section{SUMMARY}

Sewage sludge, Composted town refuse, Phosphorus, Availability, Biodegradability, Chemical extraction, Mathematical model, Kinetics.

\section{Ability of several urban wastes to supply phosphorus to crops}

We have studied 5 sewage sludges and one composted town refuse and measured their fermentability and the phosphorus extracted from them by more or less powerful reagents or taken up by the plant.

We have observed the kinetics of waste phosphorus uptake by rye-grass, with monocalcium phosphate and Thomas slag as control treatments.

By comparison with monocalcium phosphate, the relative efficiency of waste phosphorus with time can be described by the following equation that account for more than $98 \%$ of variation $E=k t^{c}$

where $\mathrm{E}=$ efficiency $(\%), \mathrm{t}=$ time of root-phosphorus source contact in days (within certain limits), $\mathrm{k}$ and $c=$ special coefficients for each organic waste.

Sludges composted with sawdust were the fastest to supply the plant with phosphorus ; next came the chemical sludges. Liming of digested sludges did not appreciably change their behaviour during our test. Finally, the plant takes up the phosphorus from composted town refuse very slowly.

We have not found any simple relation between the fermentability of wastes, phosphorus extracted by different reagents and phosphorus taken up by the plant.

\section{INTRODUCTION}

Les eaux usées des villes et des industries agro-alimentaires ne sont rejetées dans le milieu naturel qu'après avoir subi, en station d'épuration, un traitement qui a pour but de diminuer leur teneur en matières en suspension et en matières organiques à évolution rapide : dans certains cas, on produit une aération forcée qui provoque une prolifération microbienne au sein de l'eau usée, suivie d'une floculation et d'une décantation ; dans d'autres, la totalité de l'épuration résulte de processus physicochimiques engendrés par l'adjonction de réactifs : floculation, décantation, filtration, etc. Il y a toujours production de boues, qui peuvent subir elles-mêmes certains traitements permettant de les stabiliser, d'en diminuer le volume, de les « hygiéniser $"$ : digestion en anaérobiose, conditionnement chimique permettant ensuite une déshydratation mécanique, compostage après mélange avec un substrat approprié, etc. (POMMEL, 1979). 
La richesse de ces produits en phosphore, issue pour plus de la moitié des détergents, constitue un des attraits majeurs pour une valorisation agricole éventuelle; encore faut-il connaître son assimilabilité (FARDEAU et al., 1977; MOREL, 1977 ; de HAAN, 1980).

Un précédent travail (POMMEL, 1981) portant sur une boue digérée urbaine nous avait permis de mettre en évidence son action fertilisante progressive.

Dans la présente étude, nous avons entrepris d'étendre la portée de ces résultats en comparant les comportements de différents déchets organiques d'origine urbaine: boues résiduaires ayant subi différents traitements et compost d'ordures ménagères.

Bien que la majeure partie du phosphore contenu dans les boues entre dans des combinaisons de nature minćrale, nous nous sommes intéressés à la microbiologic des produits car les micro-organismes pourraient exercer une agressivité différentielle selon les formes de phosphore. Aussi avonsnous cherché à caractériser la biodégradabilité de la matière organique des déchets étudiés grâce à l'emploi de méthodes respirométriques.

La mise en œuvre d'un test utilisant le ray-grass comme extractant biologique du phosphore apporté par les déchets étudiés ou par des engrais minéraux te référence permet de déceler la cinétique de libération de cet élément.

\section{MATÉRIEL ET MÉTHODE}

Pour cette étude, nous avons retenu 5 déchets urbains différant par le niveau d'évolution de leur matière organique, ou susceptibles de posséder une phase minérale fixatrice du phosphore :

- une boue biologique de Nancy, issue de bassins d'aération, ayant subi ensuite une digestion en anaćrobiose (Bd) ;

- la même boue biologique ayant subi, en plus, un traitement au chlorure ferrique et à la chaux avant déshydratation sur filtre sous vide $(\mathrm{Bc})$;

- une boue issue d'une station d'épuration de type physicochimique (LaTeste, 33), obtenue par floculationdécantation après injection de chlorure ferrique et chaux, suivie d'un épaississement et d'une filtration sur bande (Bp) ;
- une boue compostée en mélange avec de la sciure, originaire de Nantes (Bs) ;

- un compost d'ordures ménagères de la ville de Bergerac, âgé de 6 mois $(\mathrm{Om})$.

\section{A. Caractérisation des produits étudiés}

Nous avons soumis les déchets utilisés à une analyse physicochimique classique complétée par des investigations plus poussées portant sur le phosphore et la matière organique.

Les différentes extractions de phosphore pratiquées sont présentées dans le tableau 1 ; le tableau 2 regroupe les paramètres physicochimiques concernant les produits étudiés.

\section{B. Etude respirométrique}

Les produits ont également été incubés pendant 2 semaines dans un microrespiromètre différentiel à pression constante Gilson, selon la technique de JUSTE \& DELAS (1970), JuSTE et al. (1975). Une quantité de matière sèche du produit à étudier correspondant à $20 \mathrm{mg}$ de carbone est mélangée, après broyage très fin et tamisage, à $1 \mathrm{~g}$ de sol sableux contenant 8 p. 100 de carbone. Un traitement témoin est constitué de ce même sol sans apport de source extéricure de carbone, chaque traitement étant répété 3 fois. Le mélange à incuber est humecté par $1 \mathrm{ml} \mathrm{d'eau}$ permutée au début de chaque semaine. Le gaz carbonique dégagé est absorbé par $0,2 \mathrm{ml}$ de potasse à $20 \mathrm{p}$. 100 placé dans le puits central de chaque fiole. Chaque nuit, l'atmosphère des fioles est renouvelée par ouverture du système.

\section{Test biologique}

L'expérience a été conduite avec du ray-grass (Lolium hybridum Hausskn var. "Sabrina") cultivé en chambre climatisée. Cette graminée a été choisie pour sa haute potentialité d'absorption minérale et sa rapidité de croissance qui la rend particulièrement performante pour notre test (BInet \& Thammavong, 1974 ; Binet, 1979).

$2 \mathrm{~g}$ de graines ont été semées dans des pots sans fond contenant $600 \mathrm{~g}$ de sable et recevant périodiquement les solutions nutritives dépourvues de phosphore indiquées par

TABLEAU 1

Méthodes utilisées pour l'extraction du phosphore. Methods used for phosphorus extraction.

\begin{tabular}{|c|c|c|c|}
\hline Méthode & Réactif d'extraction & $\begin{array}{c}\begin{array}{c}\text { Rapport } \\
\text { sol }\end{array} \\
\text { réactif }\end{array}$ & $\begin{array}{c}\text { Temps } \\
\text { d'agitation }\end{array}$ \\
\hline Phosphore total & $\begin{array}{l}\mathrm{HCl} 5,5 \mathrm{~N} \text { après calcination } \\
\text { à } 480^{\circ} \text { pendant } 2 \mathrm{~h}\end{array}$ & $1 / 5$ & Ebullition \\
\hline $\begin{array}{l}\text { Phosphore minéral } \\
\text { (Sommers et al., 1976) }\end{array}$ & $\mathrm{HCl} 1 \mathrm{~N}$ & $1 / 25$ & $1 \mathrm{~h}$ \\
\hline Dyer & acide citrique $2 \%$ & $1 / 5$ & 2 fois $2 h$ \\
\hline Truog & $\begin{array}{c}\mathrm{H}_{2} \mathrm{SO}_{4}, 0,002 \mathrm{~N}+ \\
\text { sulfate d'ammoniaque } 3 \%\end{array}$ & $1 / 200$ & $20 \mathrm{mn}$ \\
\hline Solution du sol & $\mathrm{CaCl}_{2} 0,01 \mathrm{M}$ & $1 / 4$ & $\begin{array}{l}2 \mathrm{~h} \text { après } 24 \mathrm{~h} \\
\text { de contact }\end{array}$ \\
\hline
\end{tabular}


TABLEAU 2

Principales caractéristiques des produits étudiés.

Main characteristics of the investigated wastes.

\begin{tabular}{|c|c|c|c|c|c|c|c|c|c|c|c|}
\hline $\begin{array}{c}\text { Caractérisation } \\
\text { des } \\
\text { produits testés }\end{array}$ & \multicolumn{2}{|c|}{$\begin{array}{l}\text { Bouc } \\
\text { digérée }\end{array}$} & \multicolumn{2}{|c|}{$\begin{array}{l}\text { Boue } \\
\text { chaulée }\end{array}$} & $\begin{array}{c}\text { Boue } \\
\text { digérée } \\
\mathrm{Ba}\end{array}$ & $\begin{array}{c}\text { Bouc } \\
\text { physico- } \\
\text { chimique } \\
\text { Bp }\end{array}$ & \multicolumn{2}{|c|}{$\begin{array}{c}\text { Boue } \\
\text { compostée } \\
\text { Bs }\end{array}$} & \multicolumn{2}{|c|}{$\begin{array}{c}\text { Ordures } \\
\text { ménagères } \\
\text { compostées } \\
\text { Om }\end{array}$} & $\begin{array}{c}\text { Scories } \\
\mathrm{S}\end{array}$ \\
\hline Humidité p. 100 & 90 & & 79 & & - & 80 & 24 & & 42 & & 50 \\
\hline pH & 6,9 & & 9,9 & & - & 8,1 & 6,4 & & 8,1 & & 12,6 \\
\hline Cendres p. 100 & 56,2 & & 60,9 & & 54,4 & 58,5 & 29,1 & & 72 & & 100 \\
\hline Carbone Anne p. 100 & 27,0 & & 24,2 & & 29,2 & - & 36,1 & & - & & traces \\
\hline Azote Kjeldahl p. 100 & 2,68 & & 2,33 & & 2,79 & 1,66 & 1,92 & & 1,03 & & traces \\
\hline $\begin{array}{l}\text { Phosphore total (P) \% } \\
\text { dont \% extractible par }\end{array}$ & 2,12 & & 1,55 & & 2,1 & 2,73 & 0,51 & & 0,4 & & 6,68 \\
\hline - $\mathrm{HCl} 1 \mathrm{~N}$ & 83 & & 87 & & & 95 & 98 & & 95 & & 92 \\
\hline - acide citrique $2 \%\left({ }^{*}\right)$ & 15 & $(4,8)$ & 0,6 & $(7,7)$ & & $(6,5)$ & 96 & $(3,3)$ & 10 & $(4,8)$ & \\
\hline - $\mathrm{H}_{2} \mathrm{SO}_{4} 0,002 \mathrm{~N}\left({ }^{*}\right)$ & 4 & $(6,4)$ & traces & $(8,42)$ & & & 57 & $(4,5)$ & 5 & (7) & traces $(8,8)$ \\
\hline - $\mathrm{CaCl}_{2} 0,01 \mathrm{M}\left({ }^{*}\right)$ & traces & $(7,1)$ & traces & $(8,8)$ & & traces $(7,3)$ & 3,9 & (5) & 0 & $(7,1)$ & $0 \quad(12,6)$ \\
\hline Potassium (K) p. 100 & 0,24 & & 0,16 & & - & 0,04 & 0,26 & & 0,35 & & traces \\
\hline Calcium p. 100 & 5,23 & & 13,4 & & 5,4 & 18 & 1,63 & & 4,82 & & 38,9 \\
\hline Magnésium p. 100 & 0,48 & & 0,42 & & 0,47 & 0,45 & 0,35 & & 0,18 & & 1,15 \\
\hline Fer p. 100 & 1,75 & & 3,39 & & 4,72 & 5,98 & 0,77 & & 3,94 & & 14,8 \\
\hline Cuivre, ppm & 212 & & 216 & & 309 & 112 & 260 & & 2640 & & traces \\
\hline Manganèse, $\mathrm{ppm}$ & 408 & & 346 & & 9250 & 719 & 532 & & 1080 & & 23300 \\
\hline Zinc, ppm & 2590 & & 1890 & & 7060 & 570 & 510 & & 3720 & & traces \\
\hline Cadmium, ppm & traces & & traces & & 33 & traces & traces & & traces & & traces \\
\hline Chrome, ppm & 336 & & 263 & & 74 & 48 & 1640 & & 536 & & 1950 \\
\hline Nickel, ppm & 81 & & 121 & & 246 & 12 & 97 & & 198 & & traces \\
\hline Plomb, ppm & 587 & & 437 & & 1310 & 79 & 152 & & 720 & & traces \\
\hline
\end{tabular}

(*) Les pH des solutions après extraction sont indiqués entre parenthèses.

(*) Extraction $\mathrm{pH}$ indicated in parentheses.

LEMAIRE (1977). Les oligo-éléments ont été apportés au moyen d'une solution commerciale contenant en quantités équilibrées et largement suffisantes les éléments : molybdène, bore, manganc̀se, cuivre, zinc et fer.

Après $20 \mathrm{j}$ de croissance, une semelle de racines se développe à la base du pot. Selon la méthode de STANFORDDe MENT (1957), chaque culture est alors posée sur un autre pot plein, de façon à ce que la semelle de racines entre en contact intime avec la surface du substrat. Ces pots inférieurs conticnnent $1 \mathrm{~kg}$ de sable, auquel a été ajouté le déchet à tester comparativement à 2 engrais minéraux phosphatés. Les 15 traitements retenus, chacun répété 4 fois, réalisent les mêmes apports quantitatifs de phosphore à 3 niveaux : 0,12 et $24 \mathrm{mg}$ par pot. Ils sont les suivants :

- $T_{0}$ - témoin, sans apport de phosphore ;

- $\mathrm{PMC}_{1}, \mathrm{PMC}_{2}$ - apport de phosphate monocalcique en dose simple et double;

$-\mathrm{S}_{1}, \mathrm{~S}_{2}$ - scories Thomas;

- $\mathrm{Bd}_{1}, \mathrm{Bd}_{2}$ - boue digérée ;

- $\mathrm{Bc}_{1}, \mathrm{Bc}_{2}$ - boue chaulée ;

- $\mathrm{Bp}_{1}, \mathrm{Bp}_{2}$ - boue physicochimique ;

- $\mathrm{Bs}_{1}, \mathrm{Bs}_{2}$ - boue compostée ;

- $\mathrm{Om}_{1}, \mathrm{Om}_{2}$ - compost d'ordures ménagères.

De plus, un pot a reçu une quantité pléthorique de phosphore en vue de donner la production maximale de matière sèche, ce qui permet de s'assurer à tout moment que la production de tous les traitements est bien limitée par la fourniture de phosphore et non par une autre déficience ou une toxicité.

Dans le but de réduire les interactions entre éléments nutritifs liées aux variations de composition des déchets, notamment l'interaction $\mathrm{N} \times \mathrm{P}$, les apports d'azote minéral réalisés en début d'expérience ont été "ajustés » en adoptant les coefficients d'assimilabilité suivants à l'azote des produits testés, tirés des données de CHAussod (1978) et de TERMAN et al. (1973) :

- boue digérée, boue chaulée

- boue physicochimique, bouc compostée $\quad: 0,10$

— compost d'ordures ménagères $\quad: 0,16$

Cette exception mise à part, les pots inférieurs ont reçu les mêmes solutions nutritives dépourvues de phosphore que les pots supérieurs. Ces applications, périodiquement renouvelées, sont destinées à couvrir très largement lès besoins de la plante en tous les éléments nutritifs à l'exclusion du phosphore.

L'apport d'cau est réalisé au moyen d'une mèche qui tapisse le fond des pots inférieurs et dont les extrémités plongent dans un réservoir d'eau: il y a donc 3 pots superposés (QUEMENER, 1968). Un tube coudé en siphon permet de contrôler de l'extćricur le niveau de l'eau dans le réservoir.

L'expérimentation a été conduite en chambre climatisée, avec $16 \mathrm{~h}$ d'éclairement par jour (15000 lux). Les conditions diurnes et nocturnes sont respectivement de $22^{\circ} \mathrm{C}$ et $75 \mathrm{p} .100$ d'humidité et $15^{\circ} \mathrm{C}$ et $95 \mathrm{p} .100$ d'humidité.

Les parties aériennes du ray-grass sont récoltées à $4 \mathrm{~cm}$ au-dessus du sol, $7 \mathrm{j}$ après le transfert des pots supéricurs sur les pots inférieurs, puis tous les $15 \mathrm{j}$ jusqu'à la $6^{\mathrm{C}}$ coupe. Elles sont ensuite séchées à $105^{\circ} \mathrm{C}$, pesées, broyées et la poudre obtenue a été minéralisée par voie sèche pour permettre une détermination des teneurs en phosphore par colorimétrie du complexe phosphomolybdique réduit par l'acide ascorbique (DUVAL, 1962). 


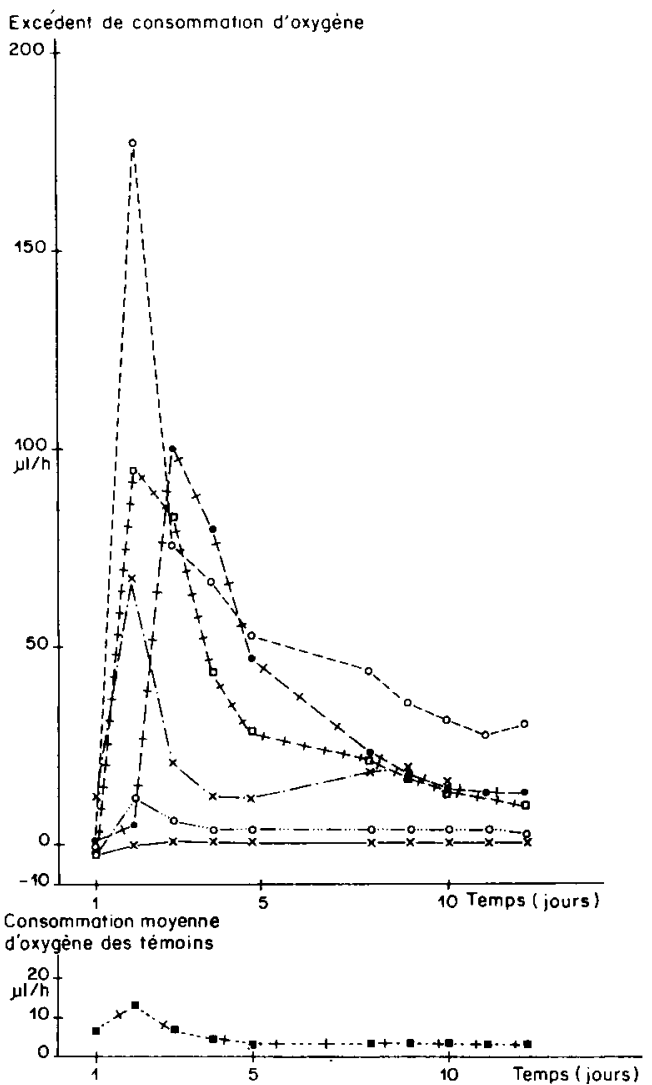

Figure 1

En bas: Evolution dans le temps de la consommation moyenne d'oxygène des échantillons de sol témoin mis en incubation.

En haut: Evolution dans le temps de l'excédent de consommation d'oxygène par rapport au témoin, selon le produit mis en incubation.

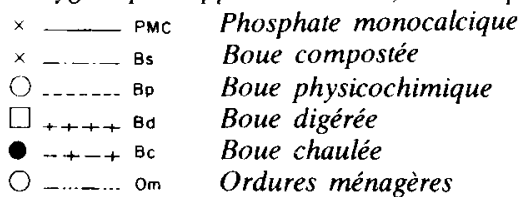

Below - Mean oxygen consumption during incubation of control soil samples.

Above - Excess oxygen consumption during incubation, according to treatments and relative to control soil samples.

\section{RÉSULTATS}

\section{A. Etude respirométrique}

La figure 1 représente l'excédent de consommation d'oxygène, relativement au témoin, des produits incubés au microrespiromètre. On observe le pic habituel de respiration des micro-organismes après 2 à $3 \mathrm{j}$ d'incubation, puis une chute, rapide d'abord, ralentie ensuite jusqu'à arriver presque à un plateau.

Dans le but de classer les produits étudiés en fonction de leur fermentescibilité, nous avons entrepris de calculer les surfaces comprises entre les portions représentatives des différentes courbes d'une part et l'axe des $\mathrm{x}$ d'autre part. Dans un souci de simplification, nous avons en effet limité notre recherche d'une formulation mathématique à la chute de la respirométrie postérieure au pic.

Comme on peut supposer que la quantité de matière fermentée et la consommation d'oxygène par unité de temps sont, à tout moment, proportionnelles à la quantité de matière fermentescible encore présente, nous avons recherché des ajustements à des exponentielles négatives et obtenu les équations suivantes :

$$
\begin{array}{lllll}
\mathrm{Q}_{(\mathrm{Bp})} & =111 & \mathrm{e}^{-0.162 t} & \text { avec } & \mathrm{r}^{2}=0,85 \\
\mathrm{Q}_{(\mathrm{Bc})}=88.2 & \mathrm{e}^{-0.245 \mathrm{t}} & \text { avec } & \mathrm{r}^{2}=0,96 \\
\mathrm{Q}_{(\mathrm{Bd})}=79 & \mathrm{e}^{-0.217 t} & \text { avec } & \mathrm{r}^{2}=0,95
\end{array}
$$

$\mathrm{Q}$ représentant la quantité d'oxygène consommée et $t$ le temps en jours, avec $t$ compris entre 0 et 9 , le temps $t=0$ correspondant aux pics de respiration. Les autres produits étudiés ont des pics de fermentescibilité moins élevés, aussi l'ajustement est-il plus lâche.

Nous avons calculé la valeur de l'intégrale de ces fonctions entre $\mathrm{t}=0$ et $\mathrm{t}=9$ et l'on obtient :

$$
\begin{aligned}
& { }_{0} \int^{9} \mathrm{Q}_{(\mathrm{Bp})}=526 \\
& { }_{0} \int^{9} \mathrm{Q}_{(\mathrm{Bc})}=320 \\
& { }_{0} \int^{9} \mathrm{Q}_{(\mathrm{Bd})}=312 .
\end{aligned}
$$

Sans vouloir préjuger du caractère de signification des Jifférences observées, on obtient le classement suivant des produits, par ordre de fermentescibilité décroissante : boue physicochimique, boue chaulée, boue digérée (Nancy), boue digérée (Ambarès), boue compostée, ordures ménagères compostécs.

On ne sera pas surpris de trouver en tête de cette liste le produit n'ayant subi aucun traitement de type biologique et, en queue, ceux qui ont déjà suivi une longue évolution aérobie.

\section{B. Test biologique}

Les exportations de phosphore par les parties aériennes du ray-grass après $7 \mathrm{j}$ de contact avec les racines et les sources de P sont représentées dans la figure 2. Les droites

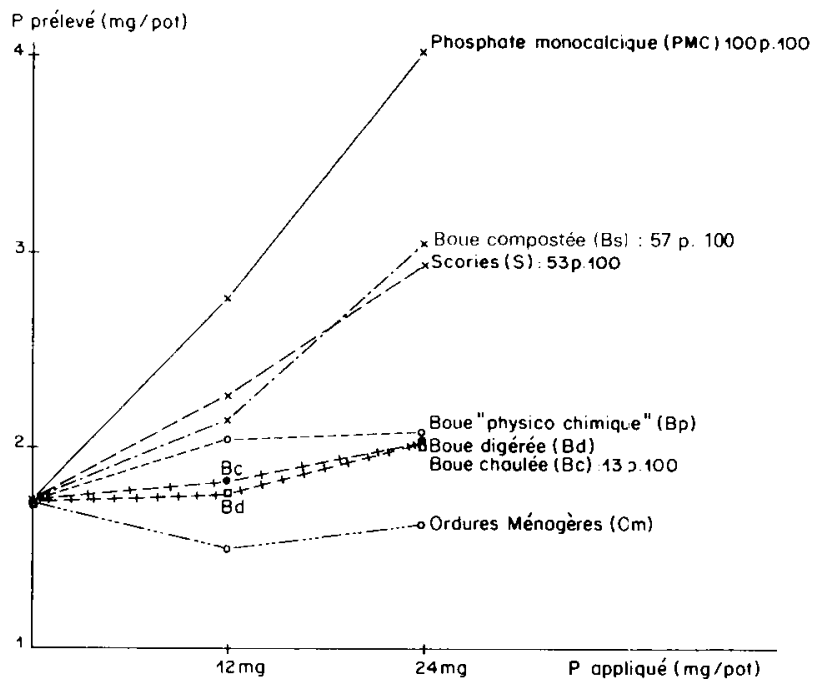

Figure 2

Exportation de phosphore par les parties aériennes du ray-grass après 7 jours de contact entre racines et sources de $P$, en fonction de la forme et de la dose d'apport. Les pourcentages représentent l'efficience du produit comme source alimentaire de $P$ relativement au phosphate monocalcique.

Uptake of phosphorus by rye-grass tops after 7 days of rootphosphorus source contact, in relation to form and dose of supply. The percentages represent the efficiency of the product as $P$ source relative to monocalcium phosphate. 
de régression des prélèvements de phosphore rapportés aux apports de cet élément ont été calculées pour chaque forme d'apport présentant un coefficient de corrélation linéaire significatif.

Par comparaison au phosphate monocalcique, l'efficience relative de chaque produit comme source alimentaire de $P$ est déterminée par le rapport des pentes des droites de régression concernant les 2 sources. On notera la supériorité de l'efficience des boues compostées et des scories par rapport à celle des autres produits testés. L'apport de compost d'ordures ménagères s'est traduit en revanche par une baisse du prélèvement de phosphore comparativement au témoin, significative dans le cas de la faible dose. On ne peut invoquer comme explication un éventuel blocage du phosphore assimilable du substrat puisque celui-ci en est dépourvu. Il s'agit donc d'un ralentissement de la translocation du phosphore de la graine vers les parties aériennes, qui pourrait s'expliquer par une colonisation plus rapide par le système racinaire du nouveau substrat qui lui est offert, ayant pour conséquence une mobilisation supérieure des réserves phosphatées à ce niveau.

Les évolutions des exportations cumulées de $\mathrm{P}$ par les parties aériennes du ray-grass sont indiquées dans les figures 3 et 4 . Elles peuvent s'ajuster à des fonctions du $2^{\text {e }}$ degré du temps et du $1^{\text {er }}$ degré en ce qui concerne la boue digérée $\mathrm{Bd}$ et les ordures ménagères Om (avec : $\mathrm{r}=0,99$ ). Les vitesses de prélèvement du phosphore sont donc des fonctions linéaires du temps du type :

$$
\text { vitesse de prélèvement }=a-b t
$$

$a$ et $b$ étant des coefficients positifs; dans le cas de la boue digérée $\mathrm{Bd}$ et des ordures ménagères $\mathrm{Om}$, le coefficient $\mathrm{b}$ est nul (fig. 5 et 6 ).

Pour chaque dose d'apport, la dimension décroissante des paramètres $\mathrm{a}$ et $\mathrm{b}$ correspondant à chaque produit permet de classer ceux-ci dans l'ordre suivant : phosphate monocalcique, scories, boue compostée avec de la sciure, boue physicochimique, boue chaulée, boue digérée, ordures ménagères compostées. Cette séquence correspond à des intensités d'alimentation décroissantes, engendrant des épuisements plus lents des apports.

L'efficience de chaque produit testé relativement au phosphate monocalcique a été calculée pour chaque coupe mais en prenant en compte le phosphore prélevé cumulé. Sa variation au cours du temps a pu être ajustée aux fonctions suivantes :

- scories : $\mathrm{E}_{(\mathrm{s})}$ $=\quad 57,1 \mathrm{t}^{0,116}$ dans l'intervalle $20<\mathrm{t}$ (jours) $<80$ avec $\mathrm{r}^{2}=\quad 0,98$

- boue compostée $E_{(B s)}=34,8 \mathfrak{t}^{0,232}$ $\begin{array}{ll}\mathrm{d}^{\circ}- & \mathrm{r}^{2}=0,99\end{array}$

- boue physicochimique $E_{(B p)}=14,2 \mathrm{t}^{0,371}$

$$
\begin{array}{ll}
-\mathrm{d}^{\circ}- & \mathrm{r}^{2}=0,98
\end{array}
$$

- boue chaulée $\mathrm{E}_{(\mathrm{Bc})}$ $-d^{\circ}$ -

$$
\begin{aligned}
\mathbf{r}^{2}=0,99 & =9,11 \mathrm{t}^{0,403} \\
& =1,76 \mathrm{t}^{0,821}
\end{aligned}
$$

- boue digérée $E_{(B d)}$ - $d^{\circ}$ -

$$
\mathrm{r}^{2}=0,99
$$

- ordures ménagères compostées $\mathrm{E}_{(\mathrm{Om})}=781 \times 10^{-5} \mathrm{t}^{1,94}$ dans l'intervalle $35<\mathrm{t}$ (jours) $<80$ avec $\mathrm{r}^{2}=0,99$.

Nous avions obtenu lors d'une étude précédente (POMMEL, 1981) portant sur une autre boue digérée :

- $\mathrm{E}_{(\mathrm{Ba})}=16,6 \mathrm{t}^{0,245}$ dans l'intervalle $20<\mathrm{t}<300$ avec $r^{2}=0,99$
Toutes ces fonctions sont représentées par des droites en coordonnées logarithmiques (fig. 7).

Si nous utilisons la symbolisation:

$$
\mathrm{E}=\mathrm{k} \mathrm{t}^{\mathrm{c}}
$$

k représentera l'efficience fictive au bout de la $1^{\text {re }}$ journée ; il s'agit donc d'un coefficient d'intensité d'alimentation phosphatée qui varie de 0 à 100 . Le paramètre $c$ indiquera la pente de la droite représentative du logarithme de l'efficience relative en fonction du logarithme du temps; ce coefficient positif traduit l'accroissement dans le temps de l'offre relative des déchets, du fait de l'épuisement plus rapide du P.M.C., et, peut-être aussi, d'une efficacité accrue de l'activité racinaire permettant l'utilisation de formes phosphatées inaccessibles dans le court terme.

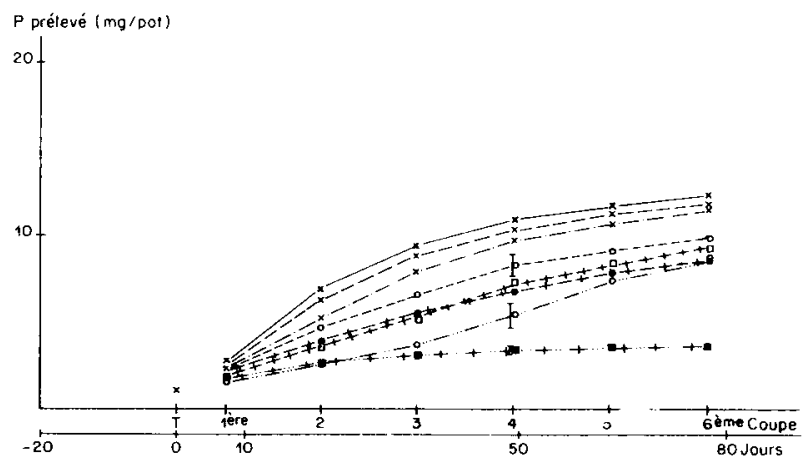

Figure 3

Exportations cumulées de phosphore par les parties aériennes du raygrass, pour les traitements ayant reçu $12 \mathrm{mg}$ de phosphore par pot, et le témoin sans apport de phosphore. Intervalles de confiance à $5 \%$. (Voir légende des symboles fig. 1.)

Cumulative uptake of phosphorus by rye-grass tops, for treatments supplied with $12 \mathrm{mg}$ phosphorus per pot, and control treatment. Confidence limits at $5 \%$.

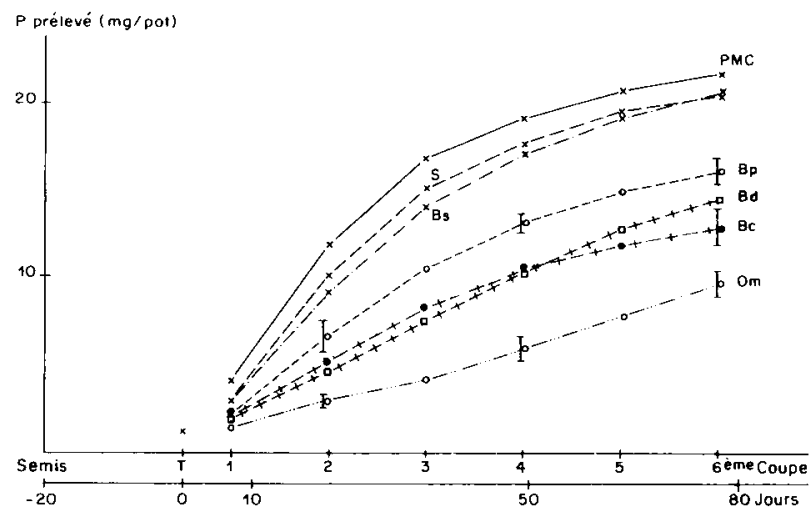

Figure 4

Exportations cumulées de phosphore par les parties aériennes du ray grass, pour les traitements ayant reçu $24 \mathrm{mg}$ de phosphore par pot. Intervalles de confiance à $5 \%$. (Voir légende des symboles fig. 1.) Cumulative uptake of phosphorus by rye-grass tops for treatments supplied with $24 \mathrm{mg}$ phosphorus per pot. Confidence limits at $5 \%$. 


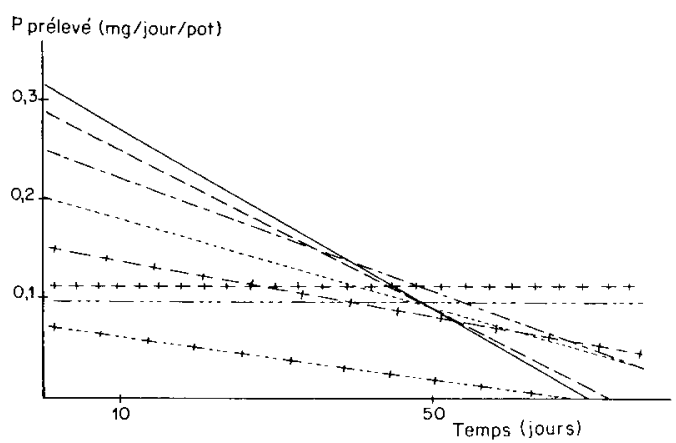

Figure 5

Vitesses d'exportation du phosphore en fonction du temps par les parties aériennes du ray-grass, pour les traitements ayant reçu $12 \mathrm{mg}$ de phosphore par pot et le témoin sans apport de phosphore. (Voir légende des symboles fig. $1+\ldots+\ldots$ Témoin sans $P$.

Rate of $P$ uptake by rye-grass tops for treatments supplied with $12 \mathrm{mg}$ phosphorus per pot, and control treatment.

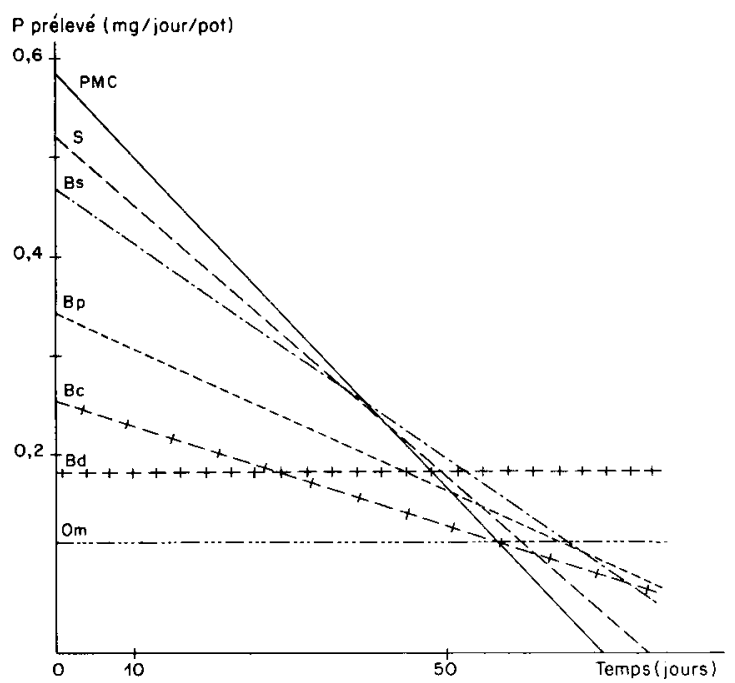

Figure 6

Vitesse d'exportation du phosphore en fonction du temps par les parties aériennes du ray-grass pour les traitements ayant reçu $24 \mathrm{mg}$ de phosphore par pot. (Voir légende des symboles fig. 1.)

Rate of $P$ uptake by rye-grass tops for treatments supplied with $24 \mathrm{mg}$ phosphorus per pot.

\section{DISCUSSION ET CONCLUSIONS}

Le phosphore des boues compostées est rapidement et presque totalement assimilable, tout comme celui des scories dans nos conditions expérimentales; ce résultat est à rapprocher des données obtenues par TAYLOR et al. (1978) sur le compost de boues et copeaux de bois préparé par aspiration forcée selon la méthode mise au point à Beltsville.

Le conditionnement de la boue de Nancy par chaulage et adjonction de fer n'a pas sensiblement modifié l'assimilabilité de son phosphore.

La boue physicochimique, très fermentescible et riche en calcium et en fer, libère mieux son phosphore que les boues digérées mais moins bien que la boue compostée, qui est la moins fermentescible du lot et la plus pauvre en calcium et en fer.

L'assimilabilité du phosphore ne semble donc pas liée de

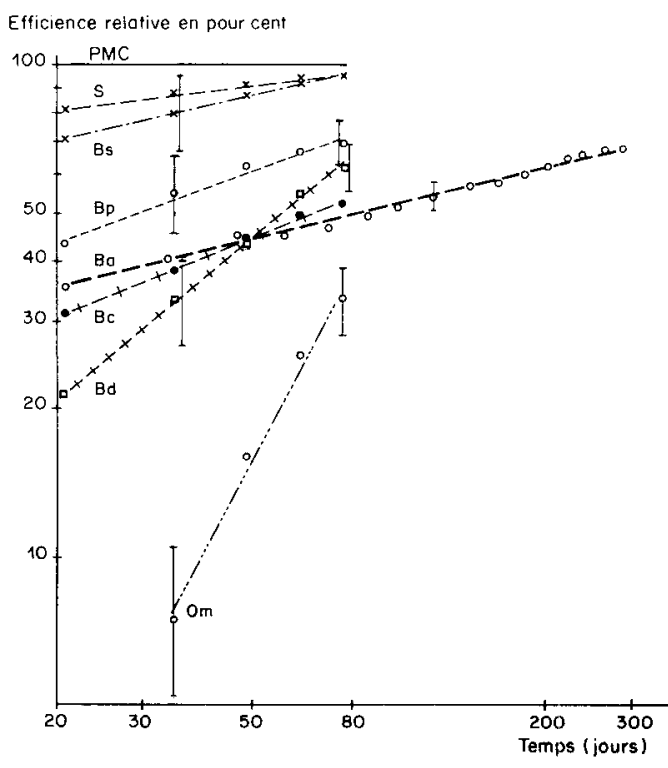

Figure 7

Efficiende relative des produits étudiés comme source alimentaire de phosphore, exprimée en pourcentage de l'effet du phosphate monocalcique et représentée en coordonnées logarithmiques en fonction du temps. Intervalles de confiance à $5 \%$. (Voir légende des symboles fig. 1. Ba: boue digérée)

Relative efficiency (\%) of waste phosphorus compared with monocalcium phosphate phosphorus on logarithmic scale. Confidence limits at $5 \%$

façon simple à la fermentescibilité de la boue ni à sa teneur en calcium et en fer.

Dans notre expérience, les boues digérées en anaérobiose ont la moindre valeur fertilisante ; il resterait néanmoins à vérifier si le caractère aérobic ou anaérobie de la décomposition des boues influe sur l'assimilabilité de leur phosphore.

Tous les réactifs chimiques utilisés extraient plus de phosphore des boues compostées que des autres boues, mais aucun ne nous en apprend véritablement plus sur le comportement de la plante.

Nous ne retrouvons pas la corrélation indiquée par GUPTA \& HÄNI (1979) entre le phosphore extrait par l'acide citrique et celui prélevé par la plante à partir de diverses boues : les différences considérables des teneurs en calcium de celles-ci perturbent probablement ce test chimique.

Quant au phosphore soluble extrait par le chlorure de calcium et que PASTENE \& COREY (données non publiées) trouvent corrélé avec le prélèvement par la plante à partir de différentes boues, il ne représente qu'une fraction très faible du phosphore disponible pour la plante et ne constitue probablement qu'un critère d'assimilabilité peu fiable.

L'extraction de phosphore par l'acide chlorhydrique reproduit le classement entre les boues obtenu à partir des réponses fournies par la plante, ce qui inciterait à penser que la plus grande assimilabilité du phosphore des boues compostées puis des boues physicochimiques pourrait être due à l'importance plus grande de la fraction minérale de cet élément. Mais ici, l'extractibilité élevée ne rend pas compte des difficultés d'alimentation de la plante, au cours des premières semaines, à partir de la plupart des sources testées.

Le phosphore contenu dans les ordures ménagères, essentiellement sous forme minérale, est fortement retenu et n'est accessible à la plante qu'après une longue phase d'activité racinaire.

En conclusion, le compostage des boues avec de la sciure 
est une opération techniquement valable, dans la mesure où elle apporte une valeur ajoutée considérable au produit, au moins dans le domaine de la fertilisation phosphatée qui fait l'objet de notre étude.

Sous ce même angle, les stations d'épuration de type physicochimique fabriquent un produit plus intéressant que les stations biologiques, mais il faudrait également prendre en compte les autres aspects du problème pour porter un jugement global: évolution du produit, minéralisation de l'azote, nuisances...

Nous n'avons pas mis en évidence, dans nos cultures sur sable, de modifications significatives résultant du chaulage des boues accompagné de l'adjonction de fer. Toutefois, un apport de boue chaulée est susceptible d'accroître le pouvoir absorbant du sol à l'ćgard des phosphates (FARDEAU et al., 1970).

Enfin, il n'est pas possible de compter sur les ordures ménagères compostées pour apporter une contribution rapide à un problème de fertilisation phosphatée. En effet, le phosphore qu'elles contiennent est peu assimilable et, du fait de la valcur élevée de lcurs rapports $\mathrm{Ca} / \mathrm{P}$ ct $\mathrm{Fe} / \mathrm{P}$, elles pourraient contribuer plus à accroître la fixation de cet élément dans le sol qu'à le fournir à la plante ; cette rétention n'est d'ailleurs pas sans intérêt dans le cas de sols à texture fortement sablcuse comme dans les Landes de Gascogne.

Reçu le 2 avril 1982. Accepté le 15 juin 1982.

\section{REMERCIEMENTS}

Nous tenons à remercier Madame BETIN, Station d'Amélioration des Plantes fourragères de l'l.N.R.A. à Lusignan, qui nous a procuré les semences utilisées dans cet essai.

\section{RÉFÉRENCES BIBLIOGRAPHIQUES}

Binet P., 1979. Extraction du potassium du sol par trois graminées prairiales, soumises à des coupes successives. Revue de la Potasse, Sect. $7, \mathbf{n}^{\circ} 4,1-5$.

Binet P., Thammavong B., 1974. Application de la méthode de DE Ment, Stanford et BradFord à l'étude de l'absorption du potassium par quelques cspèces prairiales. C. R. Acad. Agric. Fr., 1001-1009.

Chaussod R., 1978. Valeur fertilisante des boues résiduaires. Les problèmes liés à l'utilisation de l'azote. Cycle de formation permanente 18-20/4/78, E.N.S.A.I.A., Nancy.

Duval L., 1962. Dosage céruléomolybdique de l'acide phosphorique dans les sols, les végétaux et les engrais. Ann. agron., 13, 469482 .

El Baruni B., Olsen S. R., 1979. Effect of manure on solubility of phosphorus in calcareous soils. Soil Sci., 128, 4, 219-225.

Fardeau J. C., Guiraud G., Hétier J. M., 1977. Répercussions de l'épandage de boues résiduaires sur la mobilité de $\mathrm{Cd}, \mathrm{Cr}, \mathrm{Hg}, \mathrm{Zn}$ ct $\mathrm{P}$ dans les sols. - $1^{\text {cr }}$ Symp. "Recherche en matière de sols et déchets solides », Orlćans-la-Sourcc, 15-17/3/1977, Coll. RechercheEnvironnement, $\mathrm{n}^{\circ} 11$.

Fardeau J. C., Hétier J. M., Guiraud G., 1979. Etude au moyen de ${ }^{15} \mathrm{~N},{ }^{32} \mathrm{P},{ }^{65} \mathrm{Zn},{ }^{109} \mathrm{Cd}$ et ${ }^{203} \mathrm{Hg}$ de quelques limites d'utilisation en agriculture des boues résiduaires. Irst Europ. Symp. "Treatment and use of sewage sludge", Cadarachc, 13-15/2/79, 383-390.

Gupta S. K., Häni H., 1979. Estimation of available phosphate content of sewage sludge. 1rst Europ. Symp. "Treatment and use of sewage sludge", Cadarache, 13-15/2/79, 261-268.

De Haan S., 1980. Sewage sludge as a phosphate fertilizer in "Phosphorus in sewage sludge and animal waste slurries", Proc. E.E.C. Seminar, Groningen, June 12-13, 1980, 149-162.

Häni H., Gupta S. K., Furrer O. J., 1980. Availability of phosphorus fractions in sewage sludge in "Phosphorus in sewage sludge and animal waste slurries", Proc. E.E.C. Sem., Groningen, $12-13 / 6 / 80,177-190$.

Hanotiaux G., Heck J. P., Rocher M., Barideau L., MarlierGeets O., 1980. The content of $P$ in the sewage of Wallonian water purification plants and the form of $\mathrm{P}$ in these in "Phosphorus in sewage sludge and animal waste slurries", Proc. E.E.C. Sem., Groningen, 12-13/6/80, 99-108.

Juste C., Delas J., 1970. Comparaison, par une méthode respirométrique, des stabilités biologiques d'un humate de calcium et d'un humate de sodium. C. R. Acad. Sci., 270, 1127-1129.

Juste C., Delas J., Langon M., 1975. Comparaison de la stabilité biologique de différents humates métalliques. C. R. Acad. Sci. 281, $1685-1688$.

Lemaire F., 1977. Nouvelles observations sur l'appréciation de la fertilité des sols cultivés au moyen de l'expérimentation en petits vases de végétation. Ann. agron., 28 (4), 425-444.

Morel J. L., 1977. Contribution à l'étude de l'évolution des boues résiduaires dans le sol. Thèse Université de Nancy I, $117 \mathrm{p}$.

Pommel B., 1979. La valorisation agricole de déchets. 2 : Les boues résiduaires urbaines. Publication I.N.R.A., 70 p.

Pommel B., 1981. Détermination, au moyen d'un test biologique, de la cinćtique de libération du phosphore à partir d'unc boue résiduaire. Agronomie, 1 (6), 467-472.

Pommel B., 1981. A plant test-for determination of phosphorus value of urban wastes. Substrate in Horticulture. Acta Hortic. 126, 237-244.

Quemener J., 1968. Exemples d'application de la méthode de STANFORD-DE MENT à des problèmes de fertilisation phosphatée. Ann. agron., 19, 589-604.

Sommers L. E., Nelson D. W., Yost K. J., 1976. Variable nature of chemical composition of sewage sludges. J. Environ. Qual., 5, 303306 .

Stanford G., De Ment J. D., 1957. A method for measuring short term nutrient absorption by plants. I : Phosphorus. Soil Sci. Soc. Proc., 21, 612-617.

Taylor J. M., Sikora L. J., Tester C. F., Parr J. F., 1978 Decomposition of sewage sludge compost in soil : II. Phosphorus and sulfur transformations. J. Environ. Qual., 7, 119-123.

Terman G. L., Soileau J. M., Allen S. E., 1973. Municipal waste compost : effects on crop yields and nutrient content in greenhouse pot experiments. J. Environ. Qual., 2, 84-89. 